\title{
1 Epstein-Barr Virus May Contribute to Central Nervous System Involvement in HIV-positive
}

\section{Individuals}

3 Lupia $T^{l \#^{*}}$, Milia $M G^{2}$, Atzori $C^{3}$, Audagnotto $S^{1}$, Imperiale $D^{3}$, Mighetto $L^{4}$, Pirriatore $V^{1}$, Gregori $G^{2}$,

$4 \quad$ Lipani $F^{l}$, Ghisetti $V^{2}$, Bonora $S^{1}$, Di Perri $G^{l}$, Calcagno $A^{l}$.

51 Unit of Infectious Diseases, Department of Medical Sciences, University of Torino, Torino, Italy

62 Laboratory of Virology and Molecular Biology, Ospedale Amedeo di Savoia, ASL “Città di Torino”,

7 Torino, Italy

83 Unit of Neurology, Ospedale Maria Vittoria, ASL “Città di Torino”, Torino, Italy

94 Laboratory of Immunology, Ospedale Maria Vittoria, ASL “Città di Torino”, Torino, Italy.

Running Head: EBV and HIV in the CSF

\#Address correspondence to Tommaso Lupia, Tommaso.lupia89@gmail.com

*Present address: Tommaso Lupia, Unit of Infectious Diseases, Department of Medical Sciences, University of Torino, Amedeo di Savoia Hospital, C.so Svizzera 164, 10149 Torino, Italy

Type of article: Original article

Key words: EBV; HIV; central nervous system; blood brain barrier; trafficking; biomarkers; S-100 beta.

Word count: Total 2097 (Abstract 250)

\section{Figures: 3}

Tables: 2

Conflicts of Interest and Source of Funding: AC has received honoraria from Abbvie, BMS, Gilead, Janssen-Cilag, MSD, Viiv and he is currently receiving research grants from BMS, Gilead and Viiv. GDP and SB have received honoraria from Abbvie, BMS, Gilead, Janssen-Cilag, MSD, Viiv. The remaining authors have no conflict of interest to declare. 


\section{Abstract}

Epstein-Barr virus (EBV) often accesses the central nervous system (CNS) where it may lead to blood brain barrier (BBB) integrity disruption, facilitating the migration of immune cells into brain parenchyma. Our aim was to study the association between cerebrospinal fluid (CSF) EBV DNA and HIV-1 compartmental replication. $281 \mathrm{HIV}$-positive adults undergoing lumbar punctures for clinical reasons (excluding those with lymphoproliferative disorders) and CSF samples were examined. CSF virological, neurodamage (tau, p-tau, 1-42 beta amyloid) and immune activation (neopterin and S100beta) markers were measured by immune-enzymatic, ELISA and PCR validated methods. Two hundred eighty one patients were included; $111(40.5 \%)$ were naïve for antiretroviral treatment. CSF EBV DNA was detectable in $25(21.9 \%)$ naïve and $26(16 \%)$ treated patients at low levels $(<100$ and 146 copies/mL). Naïve EBV+ subjects presented higher CSF HIV RNA, biomarkers (t-tau, p-tau, neopterin) and higher rates of pleocytosis. Treated EBV+ individuals showed pleocytosis, higher CSF HIV RNA, CSF to serum albumin ratio, IgG index and neopterin. No association was observed between detectable CSF EBV DNA and the rate of CSF escape. In patients with plasma HIV RNA $<20$ copies/mL (n=97) CSF EBV DNA was detectable in 13 subjects (13.4\%) and it was associated with pleocytosis, higher CSF HIV RNA and neopterin levels. EBV DNA was detectable in a considerable proportion of HIV-positive patients and it was associated with higher levels of CSF HIV RNA and neuronal damage/inflammation biomarkers. The role of EBV reactivation in HIV-associated CNS disorders warrant further studies.

\section{Importance}

EBV is a human gamma-herpesvirus with a seroprevalence in adults approaches $95 \%$ and the pattern of clinical manifestations is very heterogeneous and varies from asymptomatic or mild viral infection to a 
61 tightly linked with several malignancies as nasopharyngeal carcinoma, Hodgkin's lymphoma and

62 Burkitt's lymphoma. HIV-infected and immunocompetent patients were both at risk of primary

63 infection and complications linked to EBV.

64 Primary tropism of EBV is for lymphocytes (type B, T and NK), epithelial, endothelial and smooth

65 muscle cells and establishes lifelong latent infection. Central nervous system could be affected by this

66 herpesvirus in primary infection and reactivation and EBV-DNA is not an uncommon finding in CSF

67 in HIV-infected population. The significance of our research is in identifying the presence of a link

68 between HIV and EBV CNS replication.

69

\section{Introduction}

71 Epstein-Barr virus (EBV) or Human Herpesvirus 4 (HHV-4) is a widely disseminated gamma

72 herpesvirus capable to persist, lifelong and asymptomatically, in a latent infection in adults [1].

73 Transmission of EBV is so efficient that by adulthood most ( $>95 \%)$ of the world's population has been

74 infected [2].

75 EBV may affect the central nervous system (CNS) and clinical manifestations were first noted in 1931

76 by Epstein and Johansen. Both primary infection and reactivation can cause neurological diseases and

77 central nervous system involvement occurs in 1 to $10 \%$ of the cases [3]. Individual state of

78 immunocompetence, age and comorbidities have been associated with the occurrence of neurological

79 complications that include: meningo-encephalites, cerebellitis, optic neuritis, cranial nerve palsy,

80 peripheral neuropathy, Alice in Wonderland syndrome, ataxia, chorea, post-infectious autoimmune

81 disorders, including Guillain-Barrè syndrome, acute disseminated encephalomyelitis (ADEM) and

82 transverse myelitis [3][4][5]. 
83 Biopsy proven vasculitis due to EBV infection have been reported: perivascular inflammatory infiltrate

84 was dominantly composed of CD3+ and CD8+ T-lymphocytes and macrophages. Some of the CD3

85 positive cells were also EBV-encoded RNA-1 (EBER1) positive, one of the two small noncoding

86 RNAs (EBER1 \& 2) found in latently EBV infected cells [6][7][8].

87 In MS patients' brains, EBV infection in B cells seems to alters the ability of B cells to process and

88 present a pathogenetically relevant myelin autoantigen and expression of higher levels of costimulatory

89 molecules than healthy controls, suggesting an enhanced APC function of B cells in MS brains, leading

to an higher autoimmune risk [9]. EBV-positive B lymphocytes count in normal human brain is very

91 low, but is shown an higher cell number in HIV infected brains. In PCR-based studies and in situ

hybridation studies were shown a detection of EBV in both lymphomatoid tissue and in pleomorphic

lymphoid infiltrates [10].

Several works have established that EBV can infect macrovascular endothelial cells in human tissue [11] [12], human brain microvessels [13] and in culture with human umbilical vein endothelial cells (HUVECs) [14] [15]. In endothelial cells with lytic reactivation of EBV were found an increase production of pro- inflammatory molecules (CCL-2 and CCL-5) and also hyper-expression of adhesion molecules on surfaces (ICAM-1 and VCAM-1) with a potential creation of an inflammatory breach through the Blood Brain Barrier (BBB) [13] [14] [15] [16]

HIV-positive patients have a higher risk of EBV-associated diseases due to reduced immune surveillance; non-Hodgkin and Burkitt's lymphomas are among AIDS-defining conditions. Highly Active Antiretroviral Treatment (HAART) has dramatically reduced the incidence of HIV-associated dementia but milder forms of cognitive impairment as well as cerebrospinal fluid (CSF) HIV escaper persist despite treatment. Beside incomplete CSF antiretrovirals' penetration/effectiveness HIV escape may be due to enhanced blood brain barrier (BBB) permeability and secondary to other concomitant 
106 infections ("secondary escape") [17]. We aimed at studying the role of CSF EBV DNA in HIV-positive 107 subjects in terms of HIV replication, BBB damage and biomarkers of neuronal damage/inflammation.

\section{Material and Methods}

109 Adult HIV-positive patients undergoing lumbar punctures for clinical reasons, were enrolled. Patients 110 with primary central nervous system lymphomas (PCNSLs), lymphoproliferative diseases (Lds) and 111 autoimmune disorders were excluded. Demographic, immunovirological, clinical and therapeutic data 112 were recorded as well as CSF features. The protocol was approved by our Ethics Committee (Comitato 113 Etico Interaziandale di Orbassano, n. 103/2015). Patients signed a written informed consent at 114 enrollment.

115 HIV RNA was measured through the real time Polymerase Chain Reaction (PCR) assay CAP/CTM 116 HIV-1 vs. 2.0 (CAP/CTM, Roche Molecular System, Branchburg,NJ, detection limit: 20 copies/mL of 117 HIV-1 RNA). EBV DNA was measured through the real time Polymerase Chain Reaction (PCR) 118 (detection limit: 100 copies/mL of EBV DNA).

119 Quantitative determination of albumin in serum and CSF was measured by Immunoturbidimetric 120 methods (AU 5800, Beckman Coulter, Brea, CA, USA). CSAR, calculated as CSF albumin $121(\mathrm{mg} / \mathrm{L}) /$ serum albumin $(\mathrm{g} / \mathrm{L})$, was used to evaluate BBB function. BBB damage definition was derived 122 from age-adjusted Reibergrams (normal if below 6.5 in patients aged $<40$ years and below 8 in patients $123>40$ years).

124 CSF total tau (t-tau), phosphorylated tau (p-tau) and $\beta$-amyloid1-42 (A $\beta 1-42)$ were measured by 125 immunoenzymatic methods (Fujirebio diagnostics, Malvern, U.S.A.) with limitsisépiof detection 126 respectively of 57, 20 and $225 \mathrm{pg} / \mathrm{ml}$. Neopterin and S100B were measured through validated ELISA 127 methods [DRG Diagnostics (Marburg, Germany) and DIAMETRA S.r.l. (Spello, Italy), respectively]. 
128 Reference values were as follows: t-tau [ $<300 \mathrm{pg} / \mathrm{mL}$ (in patients aged $21-50$ ), $<450 \mathrm{pg} / \mathrm{mL}$ (in patients 129 aged 51-70) or $<500 \mathrm{pg} / \mathrm{mL}$ in older patients], p-tau (<61 pg/mL), 1-42 beta amyloid ( $>500 \mathrm{pg} / \mathrm{mL})$, neopterin $(<1.5 \mathrm{ng} / \mathrm{mL})$ and S100B $(<380 \mathrm{pg} / \mathrm{mL})[18]$.

131 HIV RNA was measured through the real time Polymerase Chain Reaction (PCR) assay CAP/CTM 132 HIV-1 vs. 2.0 (CAP/CTM, Roche Molecular System, Branchburg, NJ, detection limit: 20 copies/mL of 133 HIV-1 RNA). EBV DNA was measured through the real time Polymerase Chain Reaction (PCR) 134 (detection limit: 150 copies/mL of EBV DNA).

135 Data were analyzed using standard statistical methods: variables were described with medians 136 [interquartile ranges (IQR) or ranges (minimum-maximum)] and they were compared using non137 parametric tests (Mann-Whitney, Kruskal-Wallis and Spearman's tests as specified in the text). Data 138 analysis was performed using PASW software version 22.0 (IBM).

\section{Results}

141 Two hundred and eighty one adult patients were included. $111(40.5 \%)$ patients were naïve for 142 combination antiretroviral treatment (cART); baseline and immune-virological characteristics, 143 stratified by cART use, are shown in Table 1. Lumbar punctures were performed before starting 144 antiretroviral treatment in naïve late presenting subjects (CD4+ T lymphocytes <100/uL) or in 145 symptomatic treated patients with cognitive disorders, headache or other neurological complaints.

146 CSF EBV DNA was detectable in 25 naïve (21.9\%) and 26 treated (16\%) patients with median values 147 of $<100(<100-234)$ and $146(<100-612)$ copies/mL respectively. Virological, neuronal damage and 148 inflammation biomarkers stratified by cART use and CSF EBV detection are shown in Table 2. 
149 Naïve patients with detectable EBV DNA had higher CSF HIV viral load (4.2 vs. $3.7 \log _{10}$ copies/mL, $150 \mathrm{p}=0.010)$; CSF to plasma HIV RNA ratios (25 vs. 4\%, p=0.025), higher rates of pleocytosis (52\% vs. 151 12.6\%, p<0.001), CSF neuronal damage biomarkers t-tau (233 vs. $114 \mathrm{pg} / \mathrm{mL}, \mathrm{p}=0.002)$, p-tau (38 vs. $15229 \mathrm{pg} / \mathrm{mL}, \mathrm{p}=0.051)$ and neopterin ( 9.25 vs. $2.02 \mathrm{ng} / \mathrm{mL}, \mathrm{p}=0.001)$ (Figure 1 and 2, above).

153 Treated patients with detectable EBV DNA had lower CD4 cell counts (136 vs. 287 cell/uL, p=0.015) 154 and higher CSF HIV RNA (2.1 vs. $<1.3 \log _{10}$ copies/mL, p<0.001), higher levels of pleocytosis (40 vs. $1558.3 \%, \mathrm{p}<0.001)$, CSAR (6.1 vs. 5.1, $\mathrm{p}=0.011), \operatorname{IgG}$ index (4.7 vs. 3.8, p=0.042) and neopterin (1.79 vs. 156 $0.81 \mathrm{ng} / \mathrm{mL}, \mathrm{p}=0.009$ ) (Figure 1 and 2, below). Conversely 1-42 beta amyloid was lower in EBV157 positive individuals ( 788 vs. $893 \mathrm{pg} / \mathrm{mL}, \mathrm{p}=0.007$ ).

158 The rate of CSF escape was similar in EBV-positive naïve (8.3\% vs. 4.5\%, p=0.607) and treated 159 patients $(28 \%$ vs. $23.3 \%, \mathrm{p}=0.615)$.

160 In plasma controllers (HIV RNA <20 copies/mL, n=97) CSF EBV DNA was detectable in 13 161 individuals (13.4\%): it was associated with pleocytosis (50\% vs. 8.4\%, p=0.001), higher CSF HIV 162 RNA in those with detectable viral load (2.28 vs. $1.85 \log _{10}$ copies/mL, p=0.011) and higher CSF 163 neopterin levels [2.81 ng/mL vs. $0.77 \mathrm{ng} / \mathrm{mL}, \mathrm{p}=0.012]$ (Figure 3). In CSF controllers (CSF HIV RNA $164<20$ copies/mL, $\mathrm{n}=84$ ) CSF EBV DNA was detectable in 10 individuals (11.9\%): it was associated with 165 pleocytosis $(66.6 \%$ vs. $6.9 \%, \mathrm{p}<0.001)$ and border-line higher CSF neopterin $(3.74$ vs. $0.78, \mathrm{p}=0.06)$ 166 (Figure 3).

\section{Discussion}

In this study EBV DNA was detectable at low concentrations in up to $21 \%$ of HIV-positive patients 
171 Such prevalence decreased in treated patients although in $13.4 \%$ of those with undetectable plasma

172 viral load EBV was still detectable and associated with worse compartmental virological and 173 immunological biomarkers.

174 Several studies reported the role of EBV in immunocompromised patients and they showed that the 175 detection of EBV DNA in the CSF is a good marker of primary central nervous system lymphoma 176 (PCNSL, with sensitivity and specificity of 70\% and 80\%) [19] [20] [21] [22]. Some studies reported 177 the development of PCNSL in HIV-infected EBV-positive patients [23] [24] [25] [26].

178 Weinberg and coll. reported in some patients the presence of pleocytosis, detectable CSF EBV DNA 179 and EBV related-mRNA supporting the hypothesis that EBV DNA is not carried by latently infected 180 inflammatory cells but from actively replicating virus [27]. Furthermore EBV affects the immune 181 system and it may enhance neuronal degeneration in chronic inflammatory conditions. [13][28]. Higher 182 rates of B-amyloid protein and neurofibrillary tangles have been observed in the brains of EBV183 positive patients diagnosed with Alzheimer's dementia as compared to controls [29]. EBV may cause a 184 sub-clinical chronic infection and facilitate inflammatory cells' trafficking through the BBB thus 185 increasing HIV entrance into the CNS. [30][31]. Our data seem to confirm this hypothesis since both naïve and treated patients present higher CSF HIV viral loads (and CSF to plasma HIV RNA ratios) and white blood cells. Additionally cART-treated patients with detectable CSF EBV DNA showed higher CSF to albumin ratios supporting a potential role in the persistence of BBB damage. The latter has been shown to be a prevalent feature of patients with dementia, to persist in some subjects despite treatment and to be associated with markers of neuronal damage and inflammation [32][33][34].

191 Hosting EBV astrocytes, and microglia, theoretically could create a wide net of cell-to-cell crosstalk 192 encouraging migration of monocytic/macrophagic line cells and modifying CNS physiological 193 homeostasis [35][36][37]. This effect may be independent from HIV control and immune system 
194 improvement: these conditions have been associated with the absence of neuronal damage and with the 195 lowest CSF concentrations of neopterin [38][39][40].

In a recent study that analyzed 108 gut biopsies collected from 19 HIV-infected and 22 HIV197 uninfectediséparticipants, CMV and EBV were detected in more than $70 \%$ of samples but more 198 commonly in HIV-positive subjects [41]. 199 replication are well-known, there is still uncertainty on the role of EBV in chronic immune activation.

200 Additionally EBV may have a role in suppressing the CNS immune system and therefore maintain an 201 incomplete T-cell mediated inflammatory response; this may be achieved through the expression of 202 viral genes encoding for proteins with immunoevasin-like function. This may translate into higher rates 203 of pleocytosis but with less inflammatory activity [42][43]. On the other hand our EBV-positive treated 204 patients showed lower CD4 counts thus suggesting that immune control may be needed in order to 205 restore a partial control on EBV low level replication.

206 Some limitations of this study should be acknowledged including the low sample size, the lack of a 207 control group and the lack of plasma EBV DNA measurements. Additionally our cohorts include 208 several patients with very low nadir CD4 cell counts and heterogeneous clinical conditions: the same 209 effect may not be observed in less advanced individuals.

210 In conclusion we reported for the first time the prevalence of EBV detection in the CSF of HIV211 positive patients without lympho-proliferative disorders. Besides we observed that naïve subjects with 212 detectable CSF EBV DNA had a higher HIV viral load and higher markers of neuronal damage and 213 inflammation; in treated individuals despite a higher HIV viral load we report a higher prevalence of 214 blood brain barrier damage, pleocytosis and immune activation. Further studies are warranted for 215 understanding the contribution of EBV to HIV-associated CNS disorders. 


\section{Tables}

\section{Table 1: Baseline characteristics}

\begin{tabular}{|l|c|c|}
\hline & Naive & Treated \\
\hline n & 111 & 170 \\
\hline Age: years & $44(37.5-48)$ & $48(42.3-56.1)$ \\
\hline Male gender: n (\%) & $78(70.7 \%)$ & $117(69.1 \%)$ \\
\hline BMI: Kg/m ${ }^{2}$ & $24.5(19,6-25,1)$ & $22.8(20-25.2)$ \\
\hline CD4: Cells/uL & $67(27-157)$ & $319(116-570)$ \\
\hline CD4 nadir: Cells/uL & $64(25-141)$ & $46(13-194)$ \\
\hline plasma VL: Log $_{10}$ copies/mL & $5.3(4.9-5.9)$ & $1.3(<1.3-1.9)$ \\
\hline CSF VL: Log $_{10}$ copies/mL & $3.9(2.9-4.7)$ & $1.4(<1.3-2.1)$ \\
\hline Time with HIV-positive test: months & $78(47-205)$ & $197(95-290)$ \\
\hline ARV classes: NNRTI & - & 43 \\
PI & - & 106 \\
INSTI & - & 45 \\
MVC & - & 16 \\
Indication for LP:Late Presentation & 48 & 49 \\
Opportunistic Infection & 24 & 32 \\
HAND & 16 & 40 \\
Syphilis & 1 & 5 \\
Follow up & 0 & 0 \\
Headache & 0 & 2 \\
\hline
\end{tabular}


CSF EBV DNA in those with detectable levels: copies/mL
$<100(<100-292)$

$26(16 \%)$

$141(<100-448)$

226 Table 1: Demographic, immunovirological and clinical variables of subjects at baseline. "BMI" body

227 mass index, "VL" viral load, "CSF" cerebrospinal fluid, "HAART" Highly Active Antiretroviral

228 Treatment, "NNRTI" non-nucleoside reverse transcriptase inhibitor, "PI" protease inhibitor, "INSTI"

229 integrase strand-transfer inhibitor, "MVC" Maraviroc, "HAND" HIV-1 associated neurocognitive

230 disorder.

231

232

233 Table 2 Cerebrospinal fluid biomarkers characteristics of the four groups

\begin{tabular}{|c|c|c|c|c|c|c|}
\hline & \multicolumn{3}{|c|}{ Naive } & \multicolumn{3}{|c|}{ Treated } \\
\hline & CSF EBV - & CSF EBV+ & $P$ value & CSF EBV- & $\mathrm{CSF} \mathrm{EBV}+$ & $P$ value \\
\hline CSF cells: $n / u L$ & $0(0-3)$ & $10(0-43)$ & $<0.001$ & $0(0-3)$ & $0(0-40)$ & $<0.001$ \\
\hline CSF pleocytosis & $12.6 \%$ & $52 \%$ & $<0.001$ & $8.6 \%$ & $40 \%$ & $<0.001$ \\
\hline CSAR & $\begin{array}{c}5.9 \\
(4-8.7) \\
\end{array}$ & $\begin{array}{c}7.2 \\
(4.1-11.2) \\
\end{array}$ & n.s. & $\begin{array}{c}5.1 \\
(3.7-7.0) \\
\end{array}$ & $\begin{array}{c}6.1 \\
(5.2-10.6) \\
\end{array}$ & 0.008 \\
\hline BBBi & $41.2 \%$ & $47.8 \%$ & n.s. & $28.3 \%$ & $45.8 \%$ & 0.090 \\
\hline IgG index & $\begin{array}{c}0.4 \\
(0.2-0.7)\end{array}$ & $\begin{array}{c}0.5 \\
(0.2-0.8)\end{array}$ & n.s. & $\begin{array}{c}0.4 \\
(0.2-0.6)\end{array}$ & $\begin{array}{c}0.5 \\
(0.3-0.8)\end{array}$ & 0.020 \\
\hline t-tau: pg/mL & $\begin{array}{c}114 \\
(67.7-202)\end{array}$ & $\begin{array}{c}233 \\
(130-334)\end{array}$ & 0.001 & $\begin{array}{c}130.1 \\
(58-212)\end{array}$ & $\begin{array}{c}62 \\
(37.5-153)\end{array}$ & n.s. \\
\hline p-tau: pg/mL & $\begin{array}{c}29 \\
(21.6-39,2)\end{array}$ & $\begin{array}{c}38 \\
(31-44.7) \\
\end{array}$ & 0.034 & $\begin{array}{c}35 \\
(25-41.8)\end{array}$ & $\begin{array}{c}28.9 \\
(21.5-37.5)\end{array}$ & n.s. \\
\hline Aß1-42: ng/mL & $\begin{array}{c}904 \\
(643-1105)\end{array}$ & $\begin{array}{c}839 \\
(584-1181)\end{array}$ & n.s. & $\begin{array}{c}893 \\
(748-1075)\end{array}$ & $\begin{array}{c}788 \\
(636-882)\end{array}$ & 0.037 \\
\hline Neopterin: ng/mL & 2.02 & 9.25 & $<0.001$ & 0.81 & 1.79 & 0.070 \\
\hline
\end{tabular}




\begin{tabular}{|l|c|c|c|c|c|c|}
\hline & $(1.1-3.8)$ & $(3.3-12.1)$ & & $(0.5-1.4)$ & $(0.6-4.3)$ & \\
\hline S100B: $\mathrm{pg} / \mathrm{mL}$ & 150.7 & $\begin{array}{c}188.4 \\
(96-279)\end{array}$ & n.s. & $\begin{array}{c}140 \\
(85-206)\end{array}$ & $\begin{array}{c}54 \\
(37.5-210)\end{array}$ & n.s. \\
\hline
\end{tabular}

234

235 Table 2: Immunoactivation and neurodamage markers, stratified according to presence or not of EBV

236 DNA on CSF and cART. "CSF” cerebrospinal fluid, "CSAR” Cerebrospinal fluid Serum Albumin

237 Ratio, "BBBi” Blood Brain Barrier impairment, "t-tau” total tau, "p-tau”phosphorylated tau, "A $\beta 1-42$ ”

238 1-42 beta amyloid, "S100B” S100 Beta. "n.s." non significant (p values >0.05).

\section{Figure 1}

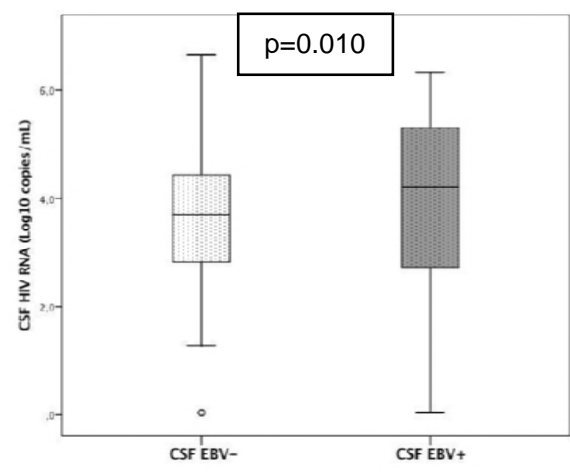

A

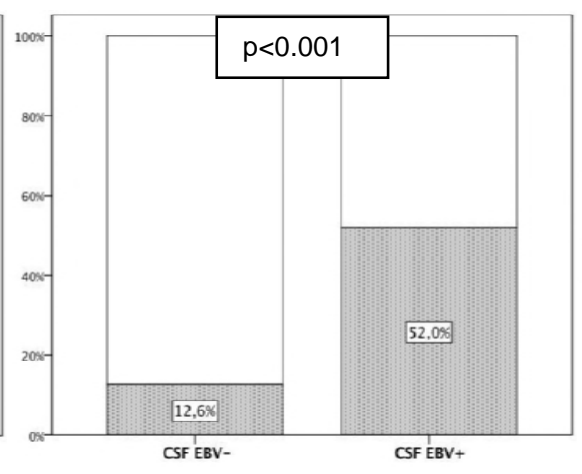

B

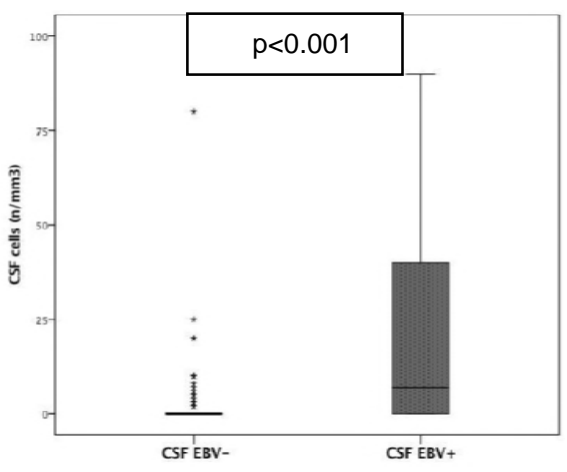

C 

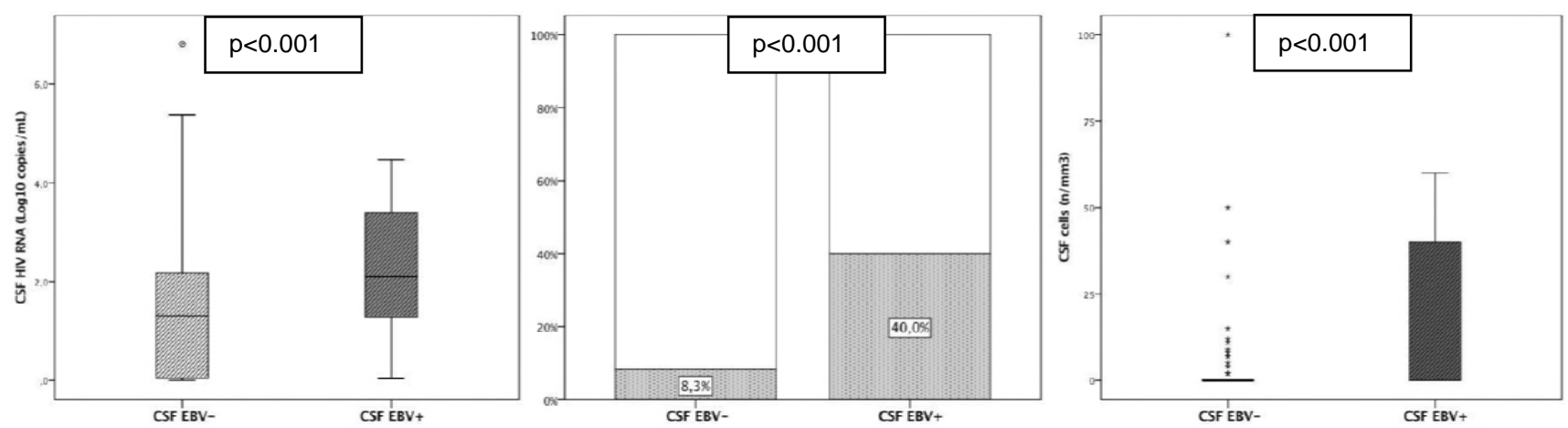

246 Figure 1: Cerebrospinal fluid HIV RNA (A), pleocytosis (B) and cell numbers (C) in naïve

247 (above) and treated patients (below). "CSF", cerebrospinal fluid; "CSF EBV+", detectable CSF EBV 248 DNA.

249

250

251

252

253 Figure 2

254

n.s.

$\mathrm{p}=0.001$

n.s.

$p=0.001$ 


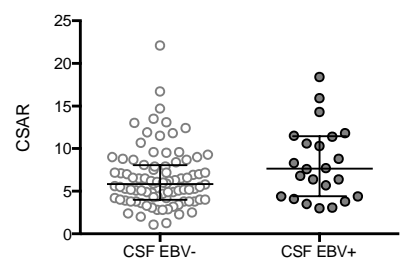

A

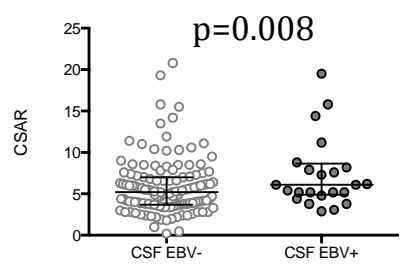

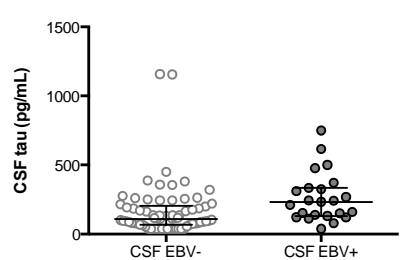

B

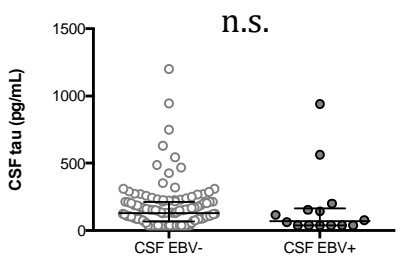

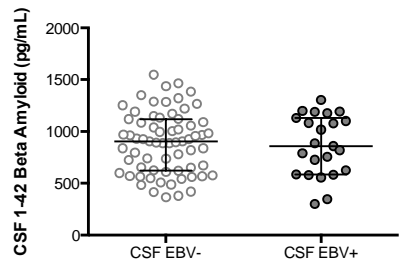

C

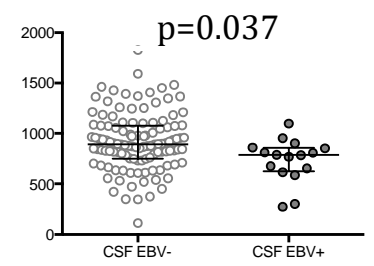

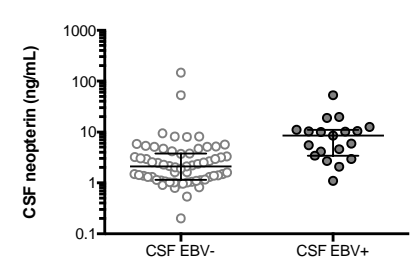

$\mathrm{D}$

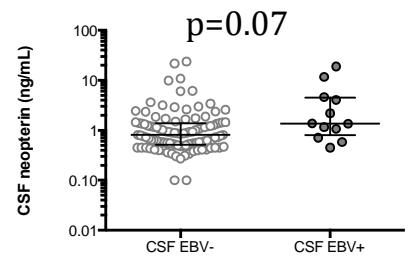

257 Figure 2: Cerebrospinal fluid biomarkers concentrations in naïve (above) and treated patients 258 (below); cerebrospinal fluid to serum albumin ratio (A), total tau (B), 1-42 beta amyloid (C) and neopterin (D). "CSF”, cerebrospinal fluid; "CSF EBV+", detectable CSF EBV DNA, "CSAR"; CSF to serum albumin ratio. All scatter dot plots present a central bar (median) with lateral error bars (IQR). In

261 all graphs white dots represent observations in the EBV-negative group (left) instead black dots picture 262 observations in EBV-positive group (right).

Figure 3 

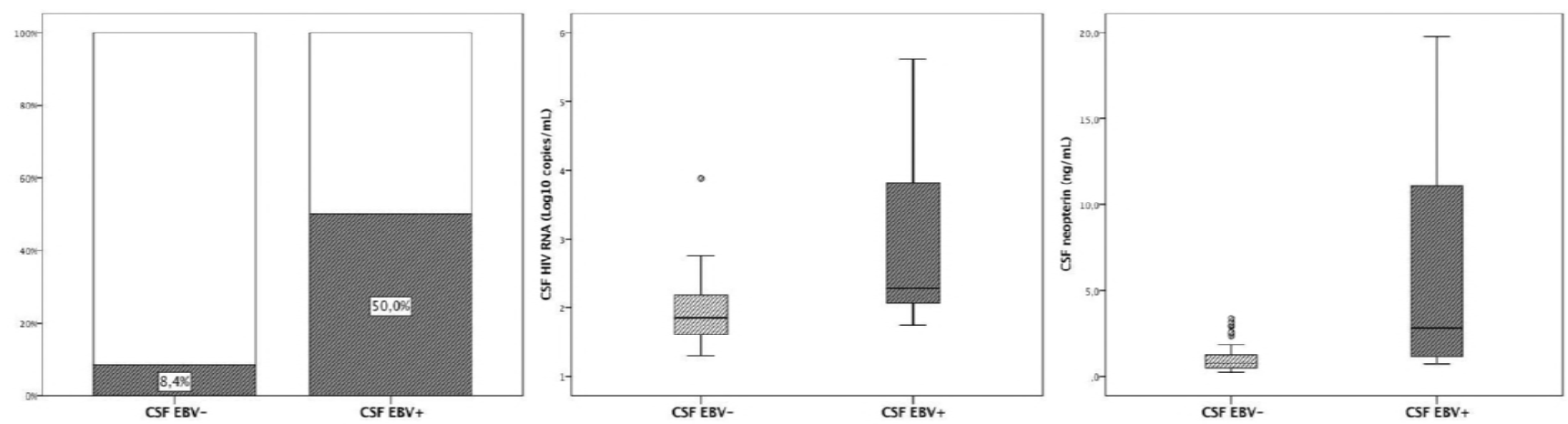

269 Figure 3: Cerebrospinal fluid pleocytosis (A), HIV RNA (B) and neopterin (C) in patients with

270 plasma HIV RNA < 20 copies/mL. "CSF", cerebrospinal fluid; "CSF EBV+", detectable CSF EBV $271 \quad$ DNA

272

273

274

275

276

277

278

279

280

281 
1. Calderwood MA, Venkatesan K, Xing L, Chase MR, Vazquez A, Holthaus AM, Ewence AE, Li N, Hirozane-Kishikawa T, Hill DE, Vidal M, Kieff E, Johannsen E (2007) Epstein-Barr virus and virus human protein interaction maps. Proc Natl Acad Sci USA 104: 7606-7611

2. Luzuriaga K, Sullivan L. Infectious mononucleosis. N Engl J Med. 2010;362:1993-2000

3. Abul-Kasim K, Palm L, Maly P, Sundgren PC. The neuroanatomic localization of Epstein-Barr virus encephalitis may be a predictive factor for its clinical outcome: a case report and review of 100 cases in 28 reports. J Child Neurol. 2009;24:720-726

4. Doja A, Bitnun A, Lee E, . Pediatric Epstein-Barr virus-associated encephalitis - 10-year review. J Child Neurol 2006; 21(5): 384-391

5. Kleinschmidt-DeMasters BK, Damek DM, Lillehei KO, Dogan A, Giannnini C. Epstein-Barr virus-associated primary CNS lymphomas in elderly patients on immunosuppressive medications. J Neouropathol Exp Neurol. 2008;67:1103-11

6. Kobayashi Z, Tsuchiya K, Takahashi M et al. An autopsy case of chronic active Epstein-Barr virus infection (CAEBV): Distribution of central nervous system (CNS) lesions. JNeurolSci2008; 275:170-7

7. Jha HC, Mehta D, Lu J, El-Naccache D, Shukla SK, Kovacsics C, Kolson D, Robertson ES. 2016. Gammaherpesvirus infection of human neuronal cells. doi:10.1128/mBio.01844-15

8. Kano K.,Katayama T., Takeguchi S., Asanome A.,Takahashi K., Saito T.,Sawada J., Saito M., Biopsy-proven case of Epstein-Barr virus (EBV)-associated vasculitis of the central nervous system, Neuropathology 2017; 37,259-264

9. Morandi E, Jagessar SA, 't Hart BA, Gran B. EBV infection empowers human B cells for autoimmunity: role of autophagy and relevance to multiple sclerosis. J Immunol (2017)

10. Anthony I. C., Crawford D. H., Bell J. E. (2003). B lymphocytes in the normal brain: contrasts with HIV-associated lymphoid infiltrates and lymphomas. Brain 126, 1058-1067

11. Ban, S., Y. Goto, K. Kamada, M. Takahama, H. Watanabe, T. Iwahori, and H. Takeuchi. 1999. Systemic granulomatous arteritis associated with Epstein-Barr virus infection. Virchows Arch. 434:249-254

12. Guarner J., Unger E.: Association of Epstein-Barr Virus in Epithelioid Angiomatosis of AIDS Patients, American Journal of Surgical Pathology. 14(10):956-960, October 1990

13. Casiraghi C, Dorovini-Zis K, Epstein-Barr virus infection of human brain microvessel endothelial cells: A novel role in multiple sclerosis, Journal of Neuroimmunology 2011 , Volume 230 , Issue $1,173-177$

14. Jones K., Rivera C., Sgadari C., Franklin J., Max E.E., Bhatia K., Tosato G. Infection of human endothelial cells with Epstein-Barr virus. J. Exp. Med. 1995;182:1213-1221

15. Xiong A, Clarke-Katzenberg RH, Valenzuela G, Izumi KM, Millan MT. Epstein-Barr virus latent membrane protein 1 activates nuclear factor-êB in human endothelial cells and inhibits apoptosis. Transplantation. 2004;78:41-9

16. Kanno H, Watabe D, Shimazu N et al. Adhesion of Epstein-Barr virus-positive natural killer cell lines to cultured endothelial cells stimulated with inflammatory cytokines. Clin Exp Immunol 2008; 151:519

17. Ferretti F., Gisslen M., Cinque P., Price RW:Cerebrospinal Fluid HIV Escape from Antiretroviral Therapy Curr HIV/AIDS Rep (2015) 12:280-288

18. Green AJ, Harvey RJ, Thompson EJ, Rossor MN (1997) Increased S100beta in the cerebrospinal fluid of patients with frontotemporal dementia. Neurosci Lett 235(1-2):5-8

19. De Luca A, Antinori A, Cingolani A, et al. Evaluation of cerebrospinal fluid EBV-DNA and IL10 as markers for in vivo diagnosis of AIDS-related primary central nervous system lymphoma. Br J Haematol. 1995;90:844-849 
20. Cinque, P, Vago L, Dahl H, et al. Polymerase chain reaction on cerebrospinal fluid for diagnosis of virus-associated opportunistic diseases of the central nervous system in HIVinfected patients. AIDS 1996;10:951-958 [i-

21. Bossolasco S, Cinque P, Ponzoni M, et al. Epstein-Barr virus DNA load in cerebrospinal fluid and plasma of patients with AIDS-related lymphoma. J Neurovirol 8: 432-438, 2002

22. Yanagisawa K., Tanuma J., Hagiwara S. et al.:Epstein-Barr Viral Load in Cerebrospinal Fluid as a Diagnostic Marker of Central Nervous System Involvement of AIDS-related Lymphoma, Intern Med 52: 955-959, 2013

23. Brink NS, Sharvell Y, Howard MR, Fox JD, Harrison MJ, Miller RF. Detection of Epstein-Barr virus and Kaposi's sarcoma-associated herpesvirus DNA in CSF from persons infected with HIV who had neurological disease. J Neurol Neurosurg Psychiatry. 1998;65:191-5

24. Cinque P, Brytting M, Vago L, et al. Epstein-Barr virus DNA in cerebrospinal fluid from patients with AIDS-related primary lymphoma of the central nervous system. Lancet 342: 398-

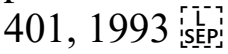

25. Rojanawiwat A, Miura T, Thaisri H, Pathipvanich P, et al. Frequent detection of Epstein-Barr virus and cytomegalovirus but not JC virus DNA in cerebrospinal fluid samples from human immunodeficiency virus-infected patients in northern Thailand. J Clin Microbiol. 2005

26. Wang J, Ozzard A, Nathan M, Atkins M, Nelson M, Gazzard B, Bower M 2007. The significance of Epstein-Barr virus detected in the cerebrospinal fluid of people with HIV infection. HIV Med 8:306-311

27. Weinberg A. , Shaobing L., Palmer M., et al: Quantitative CSF PCR in Epstein-Barr virus infection of the central nervous system. Ann Neurol 2002;52:543-548

28. Mrass P, Weninger W.. Immune cell migration as a means to control immune privilege: lessons from the CNS and tumors. Immunol Rev (2006) 213:195-212

29. Xu S., Gaskin F., Increased incidence of anti-b-amyloid autoantibodies secreted by EpsteinBarr virus transformed B cell lines from patients with Alzheimer's disease Mechanisms of Ageing and Development, 94 (1997) 213-222

30. Hutt-Fletcher LM. Epstein-Barr virus entry. Journal of Virology 2007; 81: 7825-7832

31. Houldcroft CJ, Kellam P., Host genetics of Epstein-Barr virus infection, latency and disease, Rev. Med. Virol. 2015; 25: 71-84

32. Calcagno A, Atzori C, Romito A et al (2016) Blood brain barrier impairment is associated with cerebrospinal fluid markers of neuronal damage in HIV-positive patients. J Neurovirol 22(1):88-92

33. Anesten B, Yilmaz A, Hagberg L, et al. : Blood-brain barrier integrity, intrathecal immunoactivation, and neuronal injury in HIV. Neurol Neuroimmunol Neuroinflamm. 2016;3(6):e300

34. Carroll A, Brew B. HIV-associated neurocognitive disorders: recent advances in pathogenesis, biomarkers, and treatment. F1000Res 2017; 6:312

35. A. Menet, C. Speth, C. Larcher,W. M. Prodinger,M. G. Schwendinger, P. C. et al. (1999). Epstein-Barr Virus Infection of Human Astrocyte Cell Lines. Journal of Virology, 73(9), 77227733.

36. Daugvilaite, V., Arfelt, K. N., Benned-Jensen, T., Sailer, A. W., \& Rosenkilde, M. M. (2014). Oxysterol-EBI2 signaling in immune regulation and viral infection. European Journal of Immunology, 44(7), 1904-1912. https://doi.org/10.1002/eji.201444493

37. Rutkowska, A., Preuss, I., Gessier, F., Sailer, A. W., \& Dev, K. K. (2015). EBI2 regulates intracellular signaling and migration in human astrocyte. Glia, 63(2), 341-351. https://doi.org/10.1002/glia.22757 
38. Dahl V, Low levels of HIV-1 RNA detected in the cerebrospinal fluid after up to 10 years of suppressive therapy are associated with local immune activation. AIDS. 2014;28:2251-2258

39. Chan P, Hellmuth J, Spudich S, Valcour V (2016) Cognitive impairment and persistent CNS injury in treated HIV. Curr HIV/AIDS Rep 13:209-217

40. Motta I., Allice T., Romito A., Calcagno A.:Cerebrospinal fluid viral load and neopterin in HIV-positive patients with undetectable viraemia., Antivir Ther 2017 Feb 15

41. Gianella S., Chaillon MA, Mutlu EA. : Effect of cytomegalovirus and Epstein-Barr virus replication on intestinal mucosal gene expression and microbiome composition of HIV-infected and uninfected individuals, AIDS 2017, 31:2059-2067

42. Tagawa T, et al. (2016) Epstein-Barr viral miRNAs inhibit antiviral CD4+ T-cell responses targeting IL-12 and peptide processing. J Exp Med, 10.1084/jem.2016024

43. Ressing, M.E., M. van Gent, A.M. Gram, M.J.G. Hooykaas, S.J. Piersma, and E.J.H.J. Wiertz. 2015. Immune evasion by Epstein-Barr virus. Curr. Top. Microbiol. Immunol. 391:355-381. isêpi 